\title{
Going home to die from critical care: a case study
}

\section{Article}

\section{Abstract}

Much of the activity in critical care is complex but repetitive. In order to standardise care and maintain safety, delivery of care is often directed by protocols and care bundles. This case study will reflect on an instance where care transcended the standard protocol directed path to be more individualised, creative and compassionate. Acts like these can be unique for the practitioners involved and require an element of positive risk taking, which happened here.

It will look at the decision-making, planning and risk involved in preparing for a terminally ill patient, who was inotrope and high flow oxygen dependant, to go home to have treatment withdrawn there instead of in the hospital. This was to fulfil his wish to die at home.

In unpicking the circumstances where this positive risk taking led to the desired outcome and the relationship between safety, uncertainty and risk, three themes arose. These were the journey to safe uncertainty; decision making with uncertain outcomes and the importance of robust human factors, particularly effective communication and interprofessional teamwork. If positive risk taking can result in enhanced outcomes for the patient, then the question of how this behaviour can be fostered and encouraged must be addressed.

\section{Key words \\ Safe uncertainty, Decision-making, Human factors, Critical care, End-of-life care}




\section{Introduction}

This case study will examine the process, involving positive risk taking, which resulted in a critically ill patient going home to die, as was his wish. To maintain patient confidentiality (Nursing and Midwifery Council, 2015), the patient will be referred to as Mr Smith. The case study is presented under three key headings that depict the three stages in the process - the journey to safe uncertainty; decisionmaking and uncertainty management; human factors including communication and interprofessional teamwork.

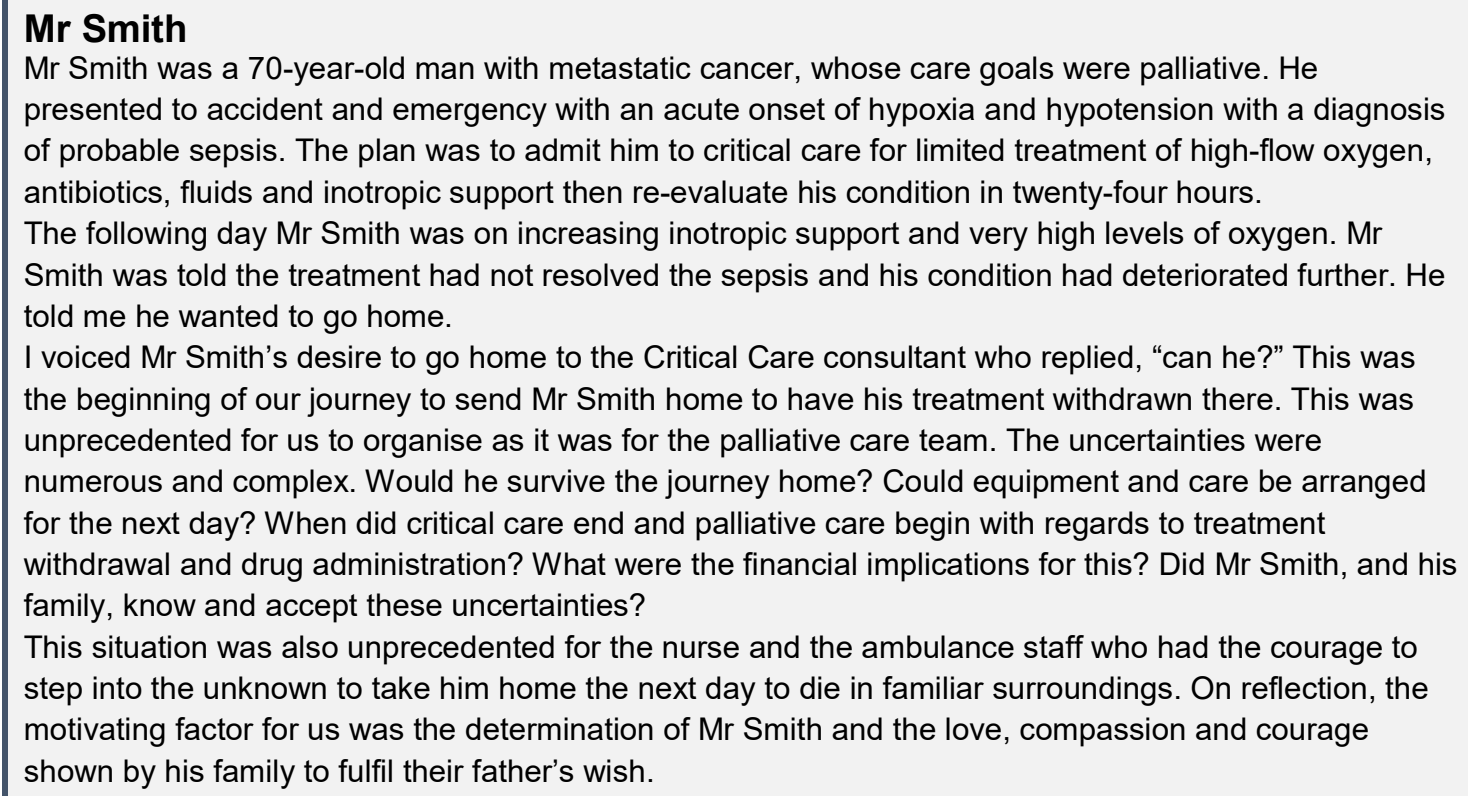

Cases involving positive risk taking have been reported, and celebrated, in the national press. Examples of this are: a critical care team arranging for a terminally ill, ventilator dependent child to go for a swim with her parents and members of the critical care team taking a patient to his son's wedding and supporting him to be there (Ford, 2016; Guys and St Thomas NHS trust, 2016). The staff in these cases talk of the planning and teamwork involved in going beyond conventional treatment to provide high-quality care. These stories resonate with the planning and teamwork involved in sending Mr Smith home to die.

Cases reporting on the discharge of critically, but also terminally ill, patients are more limited and are still rare events in the United Kingdom (Coombs et al, 2015; Coombs et al, 2017; Smith et al, $2018^{1}$ ). This is despite the fact mortality in critical care is 
relatively high and it is an accepted opinion that people would prefer to die at home (Hunter and Orlovic, 2018). Staff are generally positive about the concept of discharging critically ill patients' home to die (Lusardi et al, 2011; Coombs et al, 2015) but the complexity and unpredictability of doing so often provide too many perceived obstacles to make it feasible. This can be the patient's unstable condition, matching the patient's needs with the support available at home, financial and legal issues and variable support from family members (Lusardi et al, 2011). In the case of Mr Smith, these obstacles were encountered in varying degrees and worked through by the critical care and palliative care teams. In many other cases, this may not be possible.

Sending a patient home to die was a relatively unique event but uncertainty is a defining feature of critical care (Fackler, 2009). Therefore, uncertainty management, decision-making and effective communication are skills that are transferrable to other situations and can be developed by healthcare practitioners and healthcare educators. Shulman (2005) notes that professional education is all about linking ideas, practices and values under conditions of uncertainty. He asserts:

"In the presence of uncertainty, one is obligated to learn from experience." (p19)

\section{The journey to safe uncertainty}

The concept of safe uncertainty came from family therapy (Mason, 1993). Mason's dimensional grid reveals therapeutic positions practitioners can place themselves in when they are faced with issues of certainty and uncertainty (see table 1).

Safe certainty provides a sense of security but can over focus on the evidence base at the risk of limiting creativity (Miller and Wrate, 2014). Unsafe uncertainty is an equally rigid therapeutic position. Keeley (2009) warns that by wedding the concept of being safe to being certain, we are at risk of making ourselves unsafe by trying to control the uncontrollable. Safe uncertainty is when there is a lack of organisation and structure leading to unresolved risk factors (Miller and Wrate, 2014). Safe uncertainty is the optimum therapeutic stance where interventions are structured, but creativity is not blocked (Miller and Wrate, 2014). Safe uncertainty is not fixed, but 
always evolving (Mason, 2019). Guidelines and protocols can provide the structure to inform decision-making, but this is not the whole picture. Flexibility and creativity are also desirable when responding to uncertain situations (Mason, 2019).

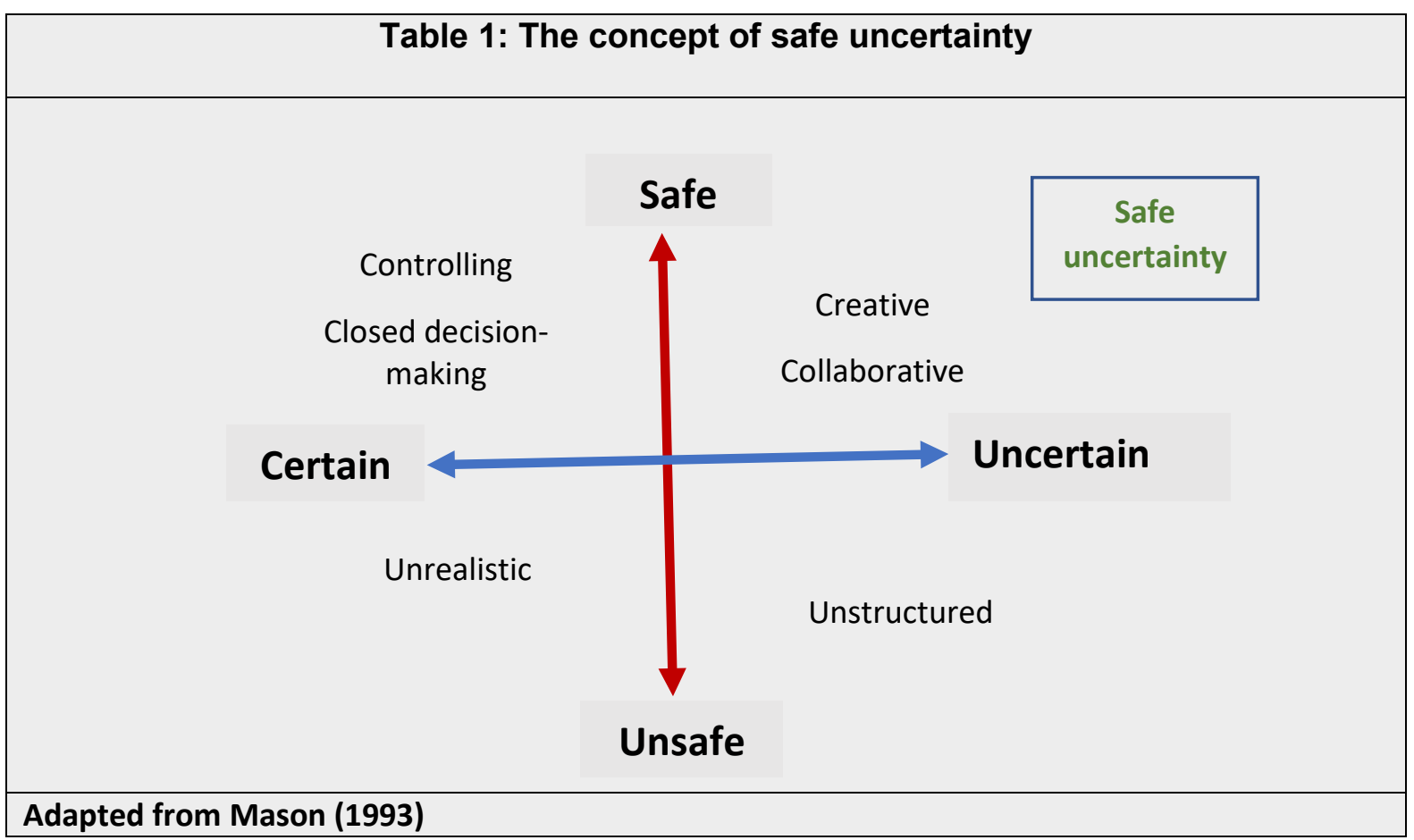

Mason (1993) defines features of working in safe uncertainty as curiosity where we do not try to understand too quickly, and solutions are not seen in absolute terms. He also advocates the position of 'authoritative doubt', where practitioners own their expertise, but in the context of uncertainty. This allows them to explore ideas in a collaborative way by being open to being influenced by others (Hardman, 2006). Keeley (2009) looked at the concept of safe uncertainty in relation to studying change management and leadership; areas he notes to have traditionally revolved around control and certainty. He included behaviours associated with different positions within Mason's framework. This concept can also be adapted to clinical practice where a position of safe uncertainty can be associated with features of creativity and collaboration (see table 1). Shulman (2005), recognised this when he observed the clinical ward rounds which he described as 'pedagogies of uncertainty'. Students at all levels were learning how to act when knowledge is limited and the outcome uncertain. Decision-making included strategies to relate to family members and what to do if the plan does not go well. Shulman (2005) acknowledges how 
experiential learning allows the practitioner to develop strategies to deal with uncertainty.

In Critical Care, patient safety is paramount. Protocols and checklists have been shown to prevent patient-safety related incidents and can improve outcomes (Bodi et al, 2017; Girbes, Robert and Marik, 2015; Day and Oxton, 2014). The objective of protocols is to provide evidence-based knowledge and ensure consistency of care (Girbes, Robert and Marik, 2015). By adopting a certain and algorithmic approach decision making can be streamlined (Rycroft-Malone et al, 2009).

The success of protocols has meant that protocolised care has mushroomed with a wide array of protocols ranging from mouthcare to sepsis. Girbes and Marik (2017) postulate the call for protocolising almost every intervention is pervasive, but protocols are best suited to simpler repetitive tasks, perhaps tasks with a more certain outcome. When moving towards uncertainty, protocols have a place, but the uniqueness of the individual means they will act in an unpredictable manner (Girbes, Robert and Marik, 2015). Simpkin and Schwartzstein (2016) note that checklists and protocols emphasise the 'black and white' aspects of medicine but do not account for the 'greyscale' of the patient's narrative. Tolerating uncertainty means the possibility of more than one answer. However safe uncertainty does involve the structure and organisation which can be provided by protocols. Coelho and Yankaskas (2017) postulate that the adoption of protocols related to end-of-life patients in the intensive care unit is fundamental. A guideline for end of life care, incorporating the potential to transfer home, could provide the structure for working in safe uncertainty. Indeed, Coombs et al (2017), noted that $20 \%$ of patients dying in critical care demonstrated potential to be transferred home. Their study gave detail about the type of patient who could be considered to transfer home to die which may be a consideration for a guideline. However, they note further consideration needs to be given to cultural and spiritual preferences and availability of healthcare resources. In planning to send $\mathrm{Mr}$ Smith home individual protocols and policies were followed (booking transport, medicines management and protocols for financial support) as no overarching end-of life guideline to include transfer home was available. They provided a necessary 
safety net and springboard to creative working and were an essential aspect for working in safe uncertainty.

Plans were explored and developed with fluidity and flexibility in communication and decision-making. Coombs et al (2015), when seeking doctors and nurse's views and experiences on transferring critically ill patient's home to die, asked them to explore three questions - should we do it? Can we do it? How do we do it? To these questions, the answer was 'I do not know' as we inhabited the greyscale of uncertainty. We needed the structure of knowing Mr Smith's, and his family's, values and expectations as well as strategies if the plan did not go as expected. This was to endeavour to make the uncertainties safe. As we were gathering information to support sending Mr Smith home, we were never certain that it was going to happen until he left Critical Care. This was uncomfortable, but it was empowering to strive to fulfil Mr Smith's wishes at the end of his life.

\section{Decision-making and uncertainty management}

Clinical decision-making is central to the role of the critical care practitioner with numerous complex and immediate decisions being made every day (LundgrénLaine, 2011). This calls for informed decision-making to ensure patient safety (Stubbing et al, 2012). These decisions concern the entire critical care process ranging from clinical to managerial decisions (Lundgrén-Laine, 2011). A defining feature of decision-making is uncertainty, if the outcome was certain then no decisions would have to be made (Muir, 2004). Fackler et al. (2009), note that decision-making in critical care is dependent on pattern recognition and in unfamiliar situations, the fragments of patterns are constructed to form a new mental model. This reorganisation of patterns to a new process was used in planning to send $\mathrm{Mr}$ Smith home. The critical care team were well rehearsed at treatment withdrawal but not in the patient's home where immediate access to other team members, medication and equipment was not available. The palliative care team were well rehearsed at providing end of life support in the patient's home but were not familiar with high flow oxygen or inotropes. Like putting the pieces of a jigsaw together to form a new picture, these pattern fragments from palliative and critical care expertise 
were integrated into a new mental model in a flexible and dynamic way. This was dependant on open communication and trust between teams.

Decision-making frameworks can be categorised as analytical, using a problemsolving approach, or intuitive, where understanding may be without rationale (Muir, 2004). Benner's seminal work looked at the influence experience had on decisionmaking among nurses (Benner, 1984). She found inexperienced nurses, or novices, relied more on policies and procedures, whereas the more experienced nurses, or experts, used intuition to inform the decision-making process. Connors and Siner (2015), when looking at decision-making in critical care, also noted that novices were more analytical and experts more intuitive in their decision-making. They found that analytical reasoning is thorough and methodical but can be slow and laborious. Intuitive reasoning relies on experience and is fast but there is a risk of early closure (Connors and Siner, 2015). They postulate that understanding different approaches to decision-making is important for educators and learners in critical care.

The clinical team planning to send Mr Smith home were all experienced therefore, it may be assumed, an intuitive approach was taken. This would have also fulfilled the need for fast paced decision-making as the situation was time critical. The need for fast paced decisions is a feature of critical care (Lundgrén-Laine, 2011; Connors and Siner, 2015). However, Mason (1983) described a feature of safe uncertainty as not finding absolute solutions. The intuitive thinker could risk closing the decision-making process too quickly which is not conducive to working in safe uncertainty. When planning to send Mr Smith home, a combination of 'intuition' and analysis was used (Connors and Siner, 2015). Although the clinical team were all experienced, the situation was unfamiliar. Price et al (2017) note that in familiar situations intuition may suffice but, if unfamiliar, systematic analysis of the situation should occur. The team intuitively knew that Mr Smith would die quickly when treatment was withdrawn due to experience, but experience also meant they knew uncertainty always prevailed when trying to predict when. Plans for him dying before he left critical care, on the journey home and if he lived for a few days involved candid conversations with his family about what may happen and planning for support and finance if he survived a longer time than expected. A hypothetical decision tree was formulated to try to decide a plan for various scenarios. 
Decision-making may involve various processes, but a key component of effective decision-making is that it is collaborative with a clear understanding of each other's roles (Lindgren- Laine,2011; Disch, 2017). This interprofessional approach involved a combination of clinical and non-clinical expertise, including legal and finance teams (see table 2).

\section{Human factors including communication and interprofessional teamwork}

Interprofessional collaboration and communication was the most important behaviour to enhance decision-making, achieve safe uncertainty and ultimately result in $\mathrm{Mr}$ Smith going home. This involved many aspects such as management of information, communication within the team and communication with the patient.

What began as a simple request to go home soon developed into a web of different communication strands as information was gathered from various sources (see table 2). Fackler et al. (2009) discuss the concept of story building, where different strands of information are combined in a meaningful way to create the patient's story. These strands were obtained from multiple sources to include medical information, tests, information about the patient's home circumstances, the finances available, legal implications and what the patient's priorities were. With so many different sources of information the challenge was to avoid fragmentation of the information strands and keep the story together. This was further complicated by the fact the story was evolving quickly and different teams had different places to document information. The patient's medical notes were a central place, so information was mainly documented there but nursing notes from both the palliative care team and the critical care team were used. On reflection, a protocol, as advocated by Coelho and Yankas (2017), involving interprofessional and standardised documentation for endof-life care would have centralised the communication strands and kept the story together in a more cohesive way. 


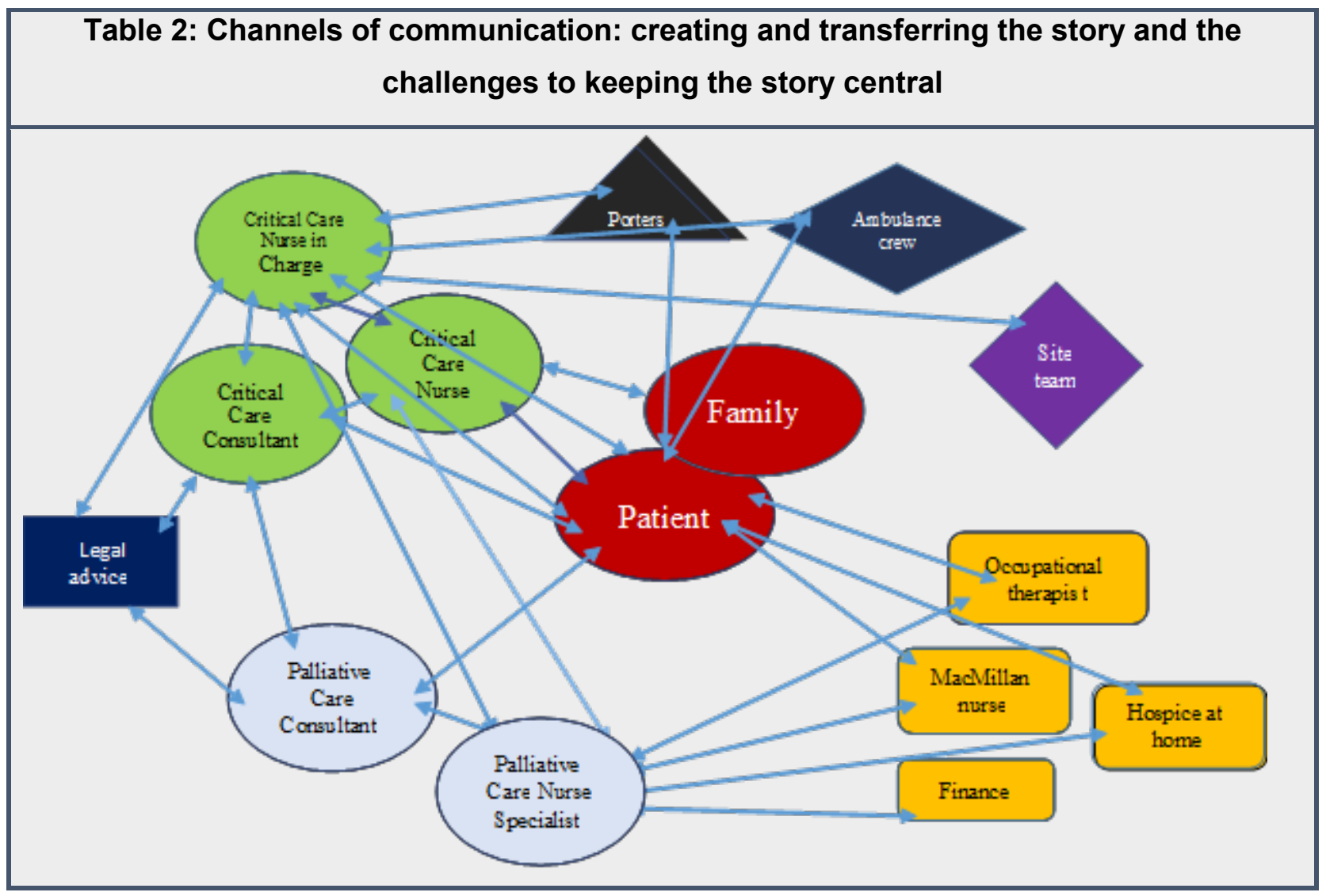

The palliative care consultant and nurse specialist came to the critical care unit to talk to the critical care team and the patient. This meant information was gathered and discussed face-to-face which made the decision-making process more efficient. It also meant all voices were heard which enhances effective team performance (Disch, 2017).

Related to story building is the activity of storytelling (Fackler et al, 2009). The information was handed over to the nurse who was going to transfer the patient home the next day as he was not involved in the preparation. Handover of care is an area of vulnerability in team communication where elements of the story can be lost (Fackler et al., 2009; Sevdalis and Brett, 2009; Disch, 2017). To mitigate this, the handover was verbal as well as written. Intuitively it was felt the complexity of the story could only be conveyed in a face-to-face discussion as well as written documentation. This also enhanced the cohesion of the team as it meant the nurse who was transferring the patient home could voice his opinions and be involved in the decision-making process. 
The team that came together for twenty-four hours from palliative care and critical care had not worked together before but a defining feature of this newly formed team from the outset was mutual respect. This was respect for the member's individual roles and expertise as well as personal courtesy. The importance of this civility, and its impact on team performance and staff wellbeing is gaining increasing recognition (Riskin, 2015; Smith et al, 2018²).

The consultants, who jointly led the team, promoted a culture where information was shared, plans adapted with new information and responsibility for tasks were allocated in a collaborative way. This was optimal team characteristics (Brindley, 2014; Disch, 2017) and, perhaps individual experience and good interprofessional relations within the separate teams resulted in this occurring.

Central to the team was the patient, Mr Smith, and his family. Heath (2016) asserts that each patient has unique values, aspirations and context. Advocating for the patient involves assessing what is most important to them and what can be done to help (Fahlberg, 2015). What was most important for Mr Smith was to die at home with his family, but communication had to be honest about the risks surrounding that and other possible outcomes. This meant adopting a position of 'authoritative doubt' (Mason, 1983). Communication with the patient's family had to be equally honest as they would provide the support for him at home and, central to him going home, was their ability to take on this extraordinary emotional task. Although family choice often drives the decision to transfer home (Coombs et al, 2017), Lusardi (2011) notes that families may not be able to fulfil this role which prevents patient's being able to go home to die. The courage shown by Mr Smith's family, in the face of so much uncertainty and risk, was the reason why Mr Smith could fulfil his wish to die at home.

\section{Implications for future practice and education}

If Shulman (2005) postulates that one learns from experience in the presence of uncertainty, Dewey (1938), in his seminal work on experiential learning, notes that the learning is dependent on the quality of the experience as well as the quality of reflection. To reflect and learn from this experience, the interprofessional team presented the case to other healthcare professionals in the hospital followed by 
questions and discussion. This also allowed other professionals to discuss and learn from the experience too. Reporting of positive cases is recognised as important in building professional behaviours and learning (Carley, 2018). As part of the discussion, a consultant opined that the positive result seemed to him luck rather than judgement. This felt disparaging of the effort and planning that went into making this happen. The emotional plea of the family and the more tacit aspects of the decision-making and teamwork were perhaps harder to convey than the policies, guidelines and analytical aspects of the process. How to make this process explicit has potential implications for educators and learners. Perhaps, as Shulman (2005) describes in 'pedagogies of uncertainty', one has to be engaged in the practice to actively learn from the experience.

The question arises if Mr Smith should have been admitted to critical care. Mirroring the complexity involved in arranging for Mr Smith to go home, decisions to admit to critical care are complex (Rees et al, 2019). With sub-optimal information and dynamic patient conditions, the decision-maker must attempt to trade the uncertainty and risk associated with various options with the most satisfactory course of action (Reader, Reddy and Brett, 2018). When Mr Smith presented to accident and emergency, the decision for limited treatment to stabilise him, alleviate symptoms and review in 24 hours was deemed the optimal course of action. This also emphasises the greyscale of decision-making.

\section{Conclusion}

The concept of working in safe uncertainty has been explored to illustrate how it can unlock curiosity and creativity leading to a real partnership between the healthcare worker and the patient. This potentially leads a good practitioner to be an excellent practitioner. The concept came from family therapy and has been applied to facilitating the transfer of a critically ill patient home to die. Recommendations for future research could look at the concept of safe uncertainty in other aspects of decision-making in critical care.

This outcome may not be reproducible because of the multiple uncertainties and the uniqueness of the situation, but the processes involving decision-making, 
communication and interprofessional working are reproducible themes to be reflected on, celebrated and repeated.

\section{References}

Benner P. (1984). From novice to expert: Excellence and power in clinical nursing practice. Menlo Park: Addison-Wesley.

Bodí M, Oliva I, Cruz Martín M, Carmen Gilavert M, Muñoz C, Olona M, Sirgo G. (2017). Impact of random safety analyses on structure, process and outcome indicators: multicentre study. Annals of Intensive Care. 7:2-11.

Brindley P. (2014). Improving teamwork in anaesthesia and critical care: many lessons still to learn. British Journal of Anaesthesia. 112: 399-401.

Carley S. (2018). Using narrative learning and story telling in Emergency Medicine. St Emlyn's. http://www.stemlynsblog.org/using-narrative-learning-and-story-telling-inemergency-medicine-st-emlyns/ [29/04/2019].

Coelho C, Yankaskas J. (2017). New concepts in palliative care in the intensive care unit. Rev Bras Ter Intensiva. 29: 222-230.

Connors G, Siner J. (2015). Clinical Reasoning and Risk in the Intensive Care Unit. Clinics in Chest Medicine. 36: 449-459.

Coombs M, Long-Sutehall T, Darlington A, Richardson A. (2015). Doctors' and nurses' views and experience of transferring patients from critical care home to die: A qualitative exploratory study. Palliative Medicine. 29:354 -362.

Coombs M, Darlington A, Long-Sutehall, Patteson N, Richardson A. (2017). Transferring patients home to die: what is the potential population in UK critical care units? BMJ Supportive \& Palliative Care; 7:98 -101. 
Day T, Oxton J. (2014). The National Early Warning Score in practice: a reflection. British Journal of Nursing. 23:1036-1040.

Dewey J. (1938). Experience and Education. New York. Touchstone.

Disch J. (2017). Teamwork and Collaboration in Quality and Safety in Nursing: A Competency Approach to Improving Outcomes $2^{\text {nd }}$ ed. Edited by Sherwood $\mathrm{G}$ and $\mathrm{J}$ Barnsteiner. New Jersey. Wiley Blackwell.

Fackler J, Watts C, Grome A, Miller T, Crandall B, Pronovost P. (2009). Critical care physician cognitive task analysis: an exploratory study. Critical Care, 13: R33.

Fahlberg B. (2015). Moral courage: A step beyond patient advocacy. Nursing. 45: $13-14$.

Ford E. (2016). "Warrior princess" Evelyn Nolan battling devastating illness receives poignant "dream swim" as parting gift. Southern Daily Echo.

http://www.dailyecho.co.uk/news/14700163.Tributes to Warrior princess who re ceived poignant dream swim as last wish/ [20/02/2019]

Girbes A, Marik P. (2017). Protocols for the obvious: Where does it start, and stop? Annals of Intensive Care. 7:1-2.

Girbes A, Robert R, Marik P. (2015). Protocols: help for improvement but beware of regression to the mean and mediocrity. Intensive Care Medicine, 41:2218-2220.

Guys and St Thomas NHS Trust newsletter. (2016). Medical team take patient to son's wedding http://www.guysandstthomas.nhs.uk/news-and-events/2016-

news/november/20161110-medical-team-take-patient-to-sons-wedding.aspx [20/02/2019].

Hardman V. (2006). Bridges to Safe Uncertainty: An Interview with Barry Mason. Australian and New Zealand Journal of Family Therapy. 27:16-21.

Heath I. (2016). How medicine has exploited rationality at the expense of humanity: an essay by lona Heath. British Medical Journal. 355: i5705.

Hunter J, Orlovic M (2018). End of life care in England. Institute for Public Policy Research, London. https://www.ippr.org/files/2018-05/end-of-life-care-in-englandmay18.pdf (accessed 20/ 02/ 2019) 
Keeley, J. (2009). Moving towards safe uncertainty: The Development of Resilience and excitement in the Future

http://www.keeleycarlisle.co.uk/Movingtowardssafeuncertainty.pdf [20/02/2019].

Lundgrén-Laine H, Kontio E, Perttilä J, Korvenranta H, Forsström J, Salanterä S. (2011). Managing daily intensive care activities: An observational study concerning ad hoc decision making of charge nurses and intensivists. Critical Care, 15:R188.

Lusardi P, Jodka P, Stambovsky M, Stadnicki B, Babb B, Plouffe D, Doubleday N, Pizlak Z, Walles K, Montonye M. (2011). The going home initiative: getting critical care patients home with hospice. Critical Care Nurse. 31:46-57.

Mason B. (1993). Towards positions of safe uncertainty. Human Systems: The Journal of Systemic Consultation and Management. 4:189-200.

Mason B. (2019). Re-visiting safe uncertainty: six perspectives for clinical practice and the assessment of risk. Journal of Family Therapy. 0:1-14

Miller L, Wrate R. (2014). Safe Uncertainty in Integrated Family Therapy. 2nd Edition. Webbish Design. http://www.integratedfamilytherapy.com/safe-uncertainty/ [20/02/2019].

Muir N. 2004. Clinical decision-making: theory and practice. Nursing Standard. 18:47-52.

Nursing and Midwifery Council. (2015). The Code: Professional standards of practice and behaviour for nurses, midwives and nursing associates. London: Nursing and Midwifery Council.

Price A, Zulkosky K, White K, Pretz J.(2017). Accuracy of intuition in clinical decision-making among novice clinicians. Journal of Advanced Nursing. 73:11471157.

Reader T, Reddy G, Brett S. (2018). Impossible decision? An investigation of risk trade-offs in the intensive care unit. Ergonomics. 61:122-133.

Rees S, Griffiths F, Bassford C, Brooke M, Fritz Z, Huang H, Rees K, Turner J, Slowther A. (2019). The experiences of health care professionals, patients, and families of the process of referral and admission to intensive care: A systematic literature review. Journal of the Intensive Care Society. 0:1-8. 
Riskin A, Erez A, Foulk T, Kugelman A, Gover A, Shoris I, Riskin K, Bamberger P. (2015). The Impact of Rudeness on Medical Team Performance: A Randomized Trial. Paediatrics. 136:487-495.

Rycroft-Malone J, Fontenla M, Seers K, Bick D. (2009). Protocol-based care: the standardisation of decision-making? Journal of Clinical Nursing, 18:1490-1500

Sevdalis N, Brett S. (2009). Improving care by understanding the way we work: human factors and behavioural science in the context of intensive care. Critical Care, 13:139.

Shulman L. (2005). Pedagogies of Uncertainty. Liberal Education. 91:18-25.

Simpkin, A, Schwartzstein R. (2016). Tolerating Uncertainty - The Next Medical Revolution? New England Journal of Medicine, 375:1713-1715.

Smith D, Hall D, Parke G, Lane R, Gray A. (2018)ํ․ No place like home: A case study of a patient discharged from an Intensive Care Unit for end of life care at home. Journal of the Intensive Care Society, 19:259-263

Smith et al, $2018^{2}$ Smith J, Morin K, Lake E. (2018) ${ }^{2}$. Association of the nurse work environment with nurse incivility in hospitals. Journal of Nursing Management, 26:219-226.

Stubbings L, Chaboyer W, McMurray A. (2012). Nurses' use of situation awareness in decision-making: an integrative review. Journal of Advanced Nursing. 68:14431453. 